\title{
Philosophy of dividing the Computer Science into Several fields.
}

\author{
Wafaa Ahmad Bazzi \\ Ph.D Student's Department of Computer Science \\ Belarusian State Technological University
}

\begin{abstract}
There are many divisions of philosophy named the philosophy of a domain where domains going from history to physics. This essay proposes some issues that might constitute the philosophy of computer science as architecture of computer, divide the science into hardware and software, programming languages, operating systems, network architecture, security, privacy, and of protect the machine by anti-virus.
\end{abstract}

Keywords: Philosophy of computer science, Hardware, Software, Programing Language, Algorithm.

\section{Philosophy of computer science introduction}

Is there relation between philosophy and computer science? "Machinery has no meaning without people and as such any research in Computer Science needs to draw upon our understanding of psychology". First of all when the scientists invent the computer, there is too much problems with calculation and static so long period of time to solve, sometimes within many years, weeks or even hours, as the result an individual must put a problem, and the machine has to solve within short period of time.Error! Reference source not found. in addition the human brain cannot store library of books or gallery of videos so there is need to construct helping memory in order to find and display some requested item. Furthermore can the built machine thinks to solve and displays the results? "The question of whether a computer can think is no more interesting than the question of whether a submarine can swim.", or is it like " if I had a computer I am sure I'd get better grades on my book reports " the machine has no brain, how it can draw a letter?, what is the result of $1+2$ ? , so if the user know to use computers ?, can he/she solve the problems by using machines in accuracy within high speed, or the consumer should study how to calculate and how to draw by using the utility, by consequence the solver must put a discipline for solution or algorithm, moreover a process or an operation of the chart which is written by English language, is it understandable by the computer, as result the computer languages to communicate with, besides that the sciences of computer is divided into two big divisions Hardware to built the device and Software to use the machine, furthermore several sciences are invented from them.

"Machinery has no meaning without people and as such any research in Computer Science needs to draw upon our understanding of psychology." $\left[{ }^{10}\right]$ 


\section{Hardware and Software}

In the computer there two sciences or two big divisions, Hardware and Software ... so what is Hardware... Hardware refers to the material parts of the machine ... some examples of Hardware are Keyboard , Screen , CPU and Mouse... is the Hardware act and produce a result without human or human logic such as how to perform a task, the first electronically machine is only for scientists even for nuclear jobs and not for usual uses at life ...moreover it is very big machinery formed with several relays need numerous of workers which work together to complete a task and as result it is hard and stressed to do as in follow Figure 1.

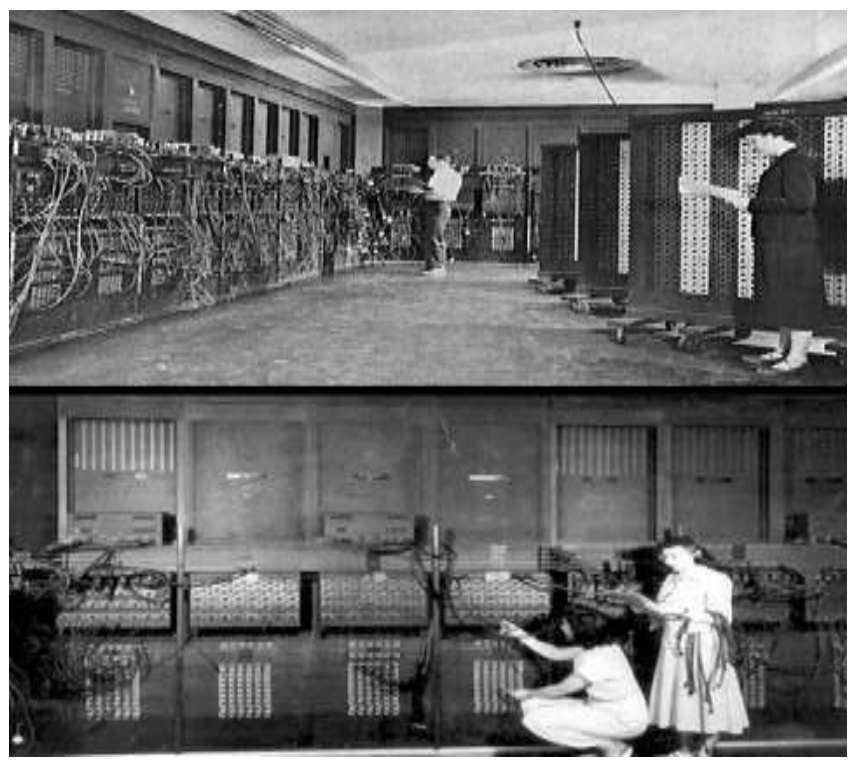

Figure 1. Early big machine

\section{Disks and Memory}

additionally the human cannot memories library of books so the thinking of constructing a helping memory by the inventor Charles Babbage who is the father of computer, he uses only the numbers to solve the problems and it is another difficulty not only with data but also with latency time taking by resolution, by consequence and with the aide of Ada Lovelace in the below Figure 2 who is the first programmer or the mother of new computer and she makes up the punched card as in the below Figure 3, so the analyzer can manipulate

- The representation of the data and not only for numbers.

- The manipulation of symbol and not only for calculation.

- The computer is used for graphical and not just for computation. 


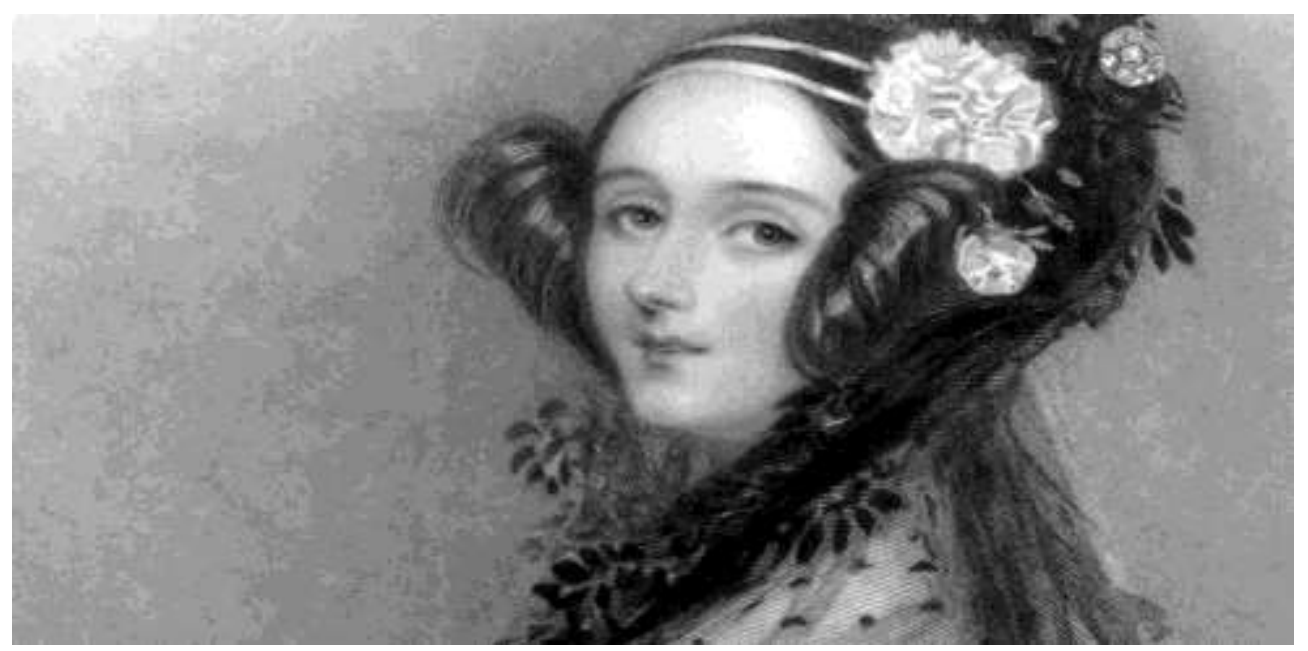

Figure 2. Lady Ada

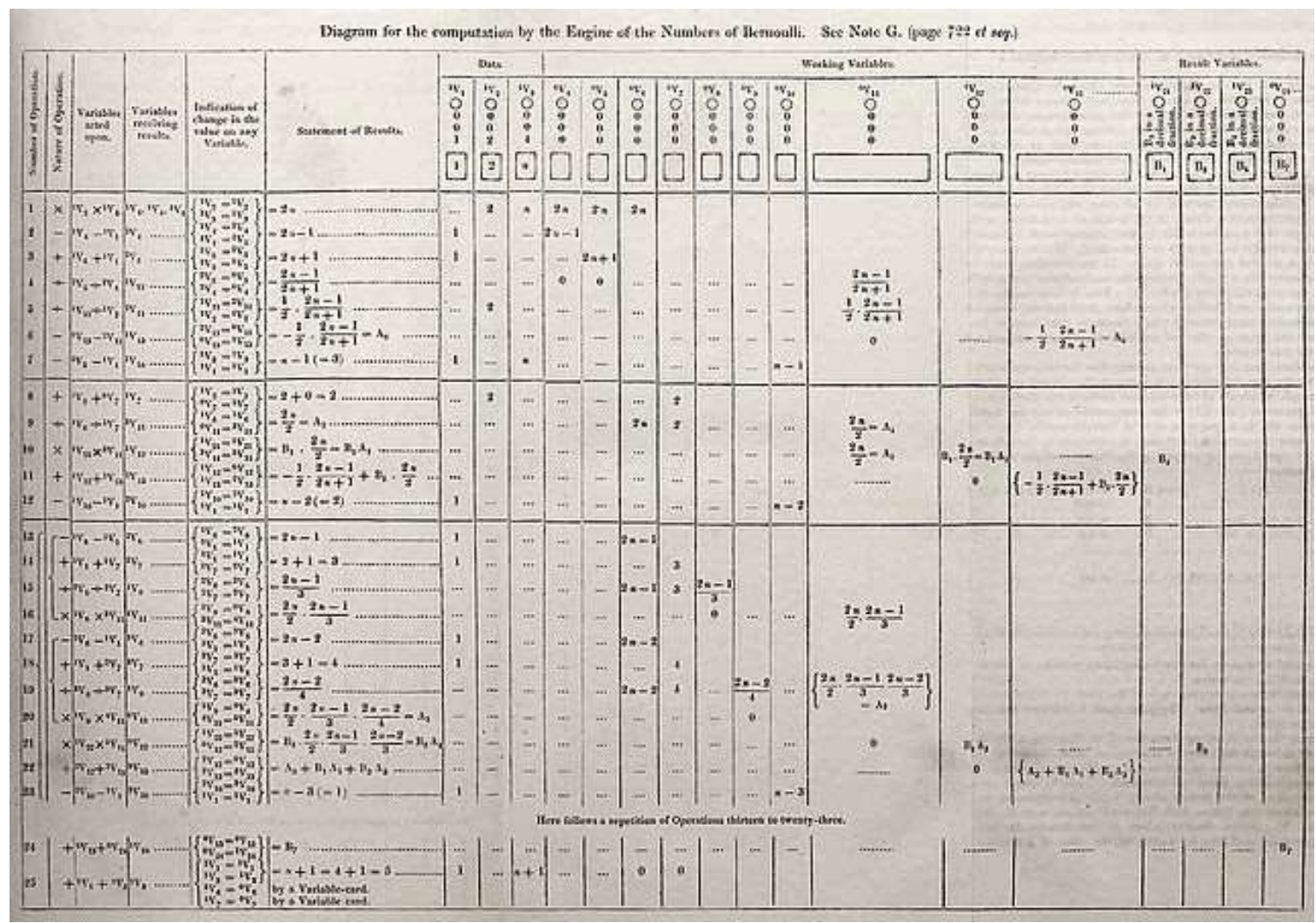

Figure 3. Punched Card

By consequence in new world of computer science begins and the century of computer language starts. Take an example in 1943 when the developers invent the one of the first computer machines which named ENIAC and which cover up over 1,800 square feet, collected in 1800 tubes and weighed at 50 tones as in the below Figure 4. 


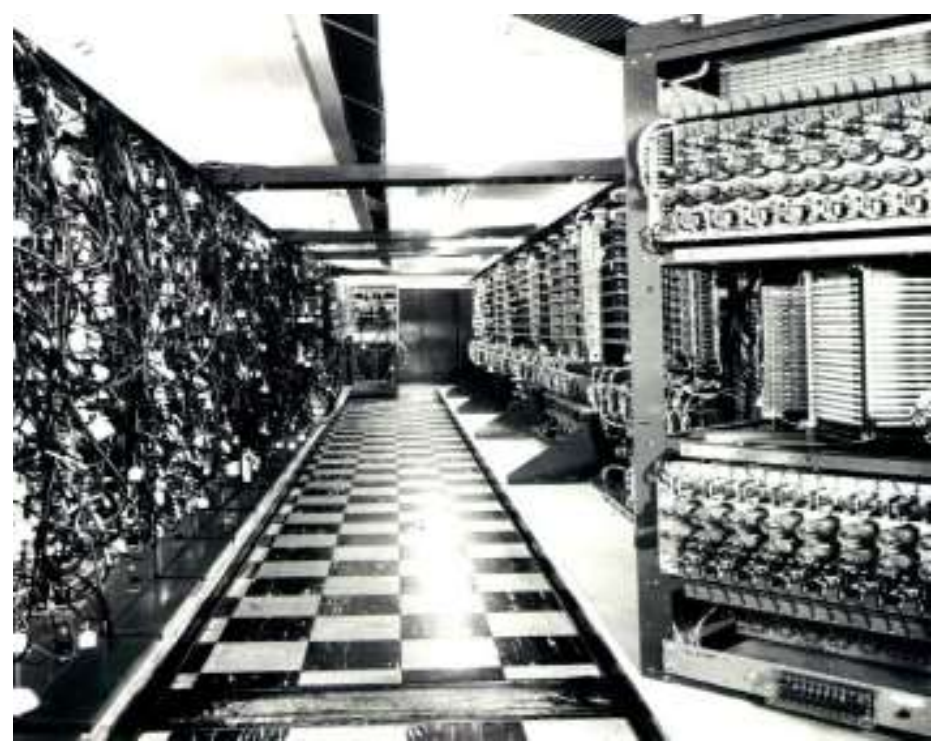

Figure 4. EINAC

It was for US Army and has only two missions it costs $400,000 \$ \ldots$ some of its functions are doing calculation of a hydrogen bomb even though it has general purpose it is programmed manually by plugging cables and putting switches and data was input on punched cards. The tasks require from several days to an hour ...the computer still as textbook to teach, so it has the knowledge base, is it enough to learn ??? Surely no, the required information must be understandable, the comprehensible need some of philosophy, and which is look like the assembly of all obtained information and extract new creative mind which is considered necessary for our life.

\section{Algorithm}

The study above tell us that need some creativity to use the invented computer in order to extract some data and produce results... as consequence require plan to tell the machine what to do.... Hence in definition computer machine solution signifies the Hardware using by a corresponding Algorithm to achieve some accurate fast results, the model of computing as in the below Figure 5.

"Computer means the Hardware, their algorithm and all that goes along with them". Error! Reference source not found.

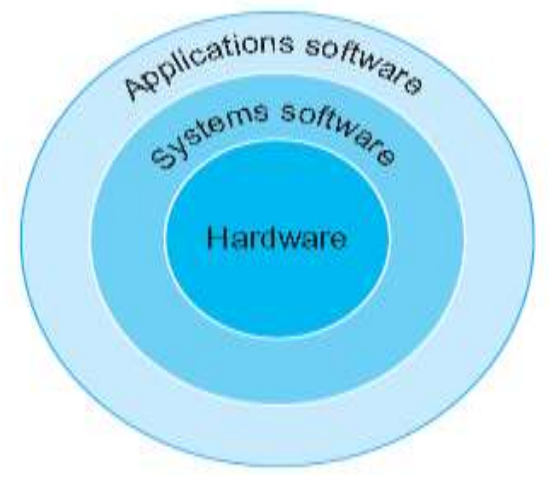

Figure 5. model of computing

\section{Software}

At the first of Invent of word software the term function was the set of manual instructions to direct the Hardware ,after that a big evaluation made by the women inventor ADA in 1880 , and to help Jacquard Loom machine and the using of string punched card to feed into machine ${ }^{[4]}$, the goal is to write commands in order to control the built machine, so with the machine Example Z1 in 1941 which is invented by Konrad 
Zuse and to reprogram ,they use the Plan Calculas, the first algorithmic programming Language,for creating solution of problems, and it is binary digital computer ${ }^{[4]}$.

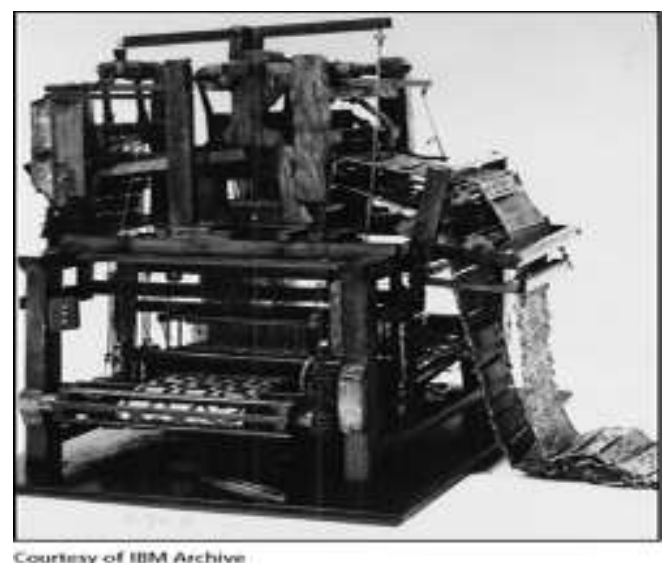

Figure 6. ${ }^{[4]}$ IBM Machine

Think about represent a program in binary system so producing of millions of bits to be translated. By consequence the scientists use the decimal take as example the ENIAC which is controlled by using 10 decimal digitals instead of binary ones, to assemble this device the inventors utilized more than 18,000 vacuum tubes as in the below Figure 8 and required to keep cool over 167 square meters (1800 square feet) of floor space, also it had punched-card to input and output.

Until 1890 the machines still mechanic, specific to a task, heavy, require multi user, and expensive, so there is solution by enhanced evaluated one, besides another creation by an American Herman Hollerith, and prior to this invention it took eight years to make static related to the habitant in the United States and fill information about. This Tabulating Machine used motor to move the data entry card and read automatically so several cards read instead of one. Approximately 65 cards could be passed through this computer in a minute $\left[{ }^{5}\right]$, below in the Figure 6 some of the first sciences inventors.

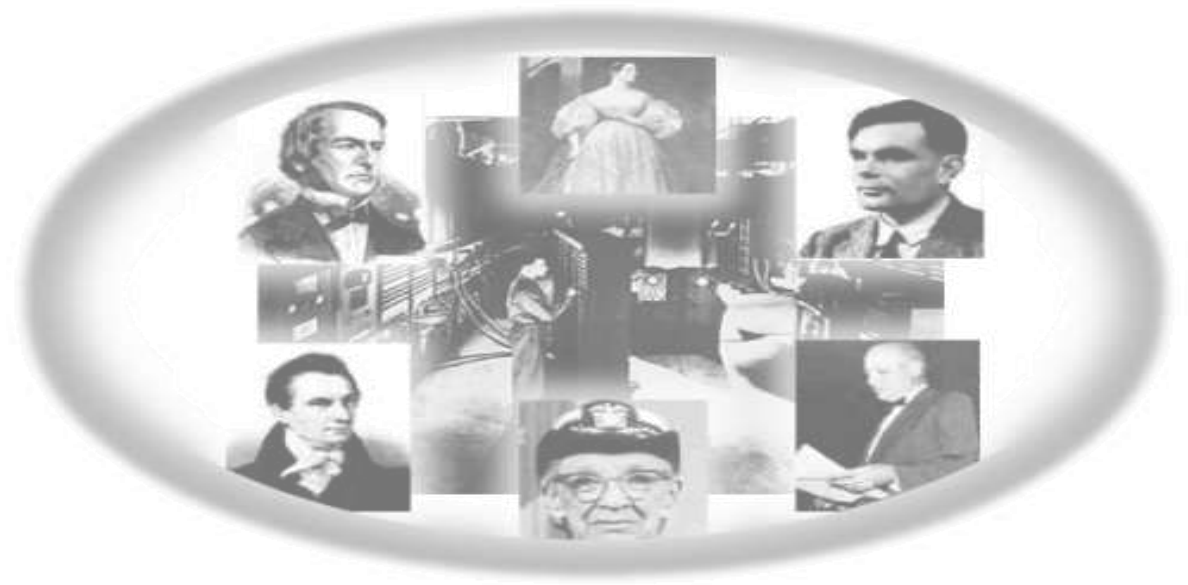

Figure $7\left[^{5}\right]$ the early inventors. 


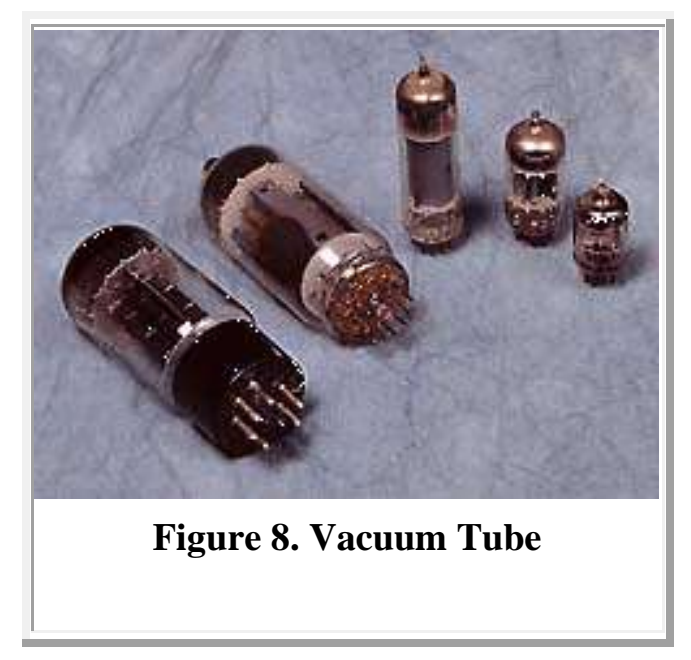

\section{Hardware innovation}

In 1950's several devices developed, that are as enhancement in computer field and the launching of the electronic computer innovation, the first of these devices are built with transistor shown in the below Figure 9, which is invented in Bell Labs in 1947, the transistor was finished the century of using vacuum tubes , radios, and the new brand become more usable for other than the scientists.

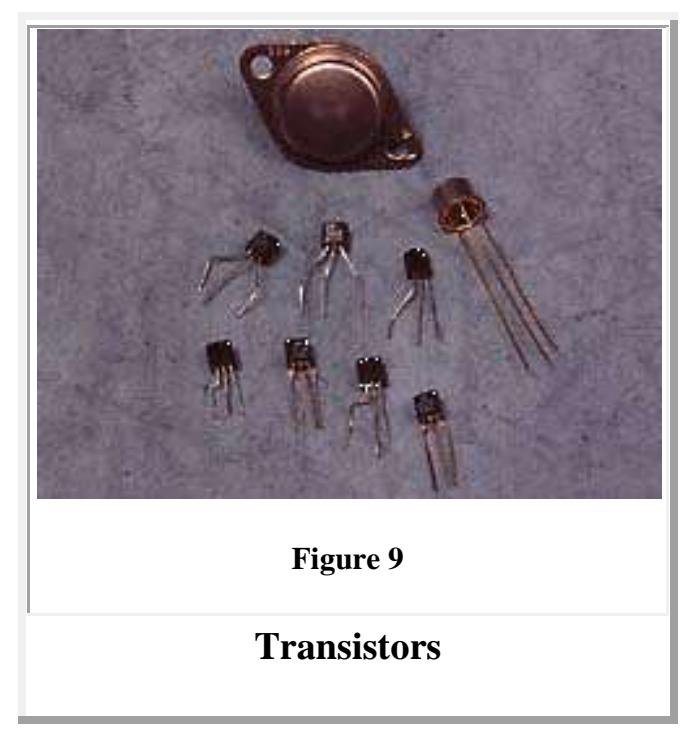

In 1958, this problem too was solved, when manufactory of the integrated circuit or chip. A chip is a collection of tiny transistors which are related together. Currently only connections were needed to other electronic components. In addition to saving space, the speed of the machine was now increased, since there was a reduced distance that the electrons follow.

\section{Philosophy of Digital world ${ }^{[6]}$}

Why there is digital world? And why all kind of information must be converted to this digital system?

Digital philosophy is a new of thinking about the basics of operations in nature. DP is an atomic theory carried to the extreme where all measures in nature are finite and discrete. This signifies that, theoretically, any amount could be represented exactly by an integer. 
Further, DP suggests that nature representation no infinities, continuities, or locally determined random variables.

At the most fundamental levels of physics, DP suggests a totally discrete process called Digital Mechanics. Digital Mechanics (DM) must be a substrate for Quantum Mechanics. Digital Philosophy makes sense with regard to any system if the following assumptions are true:

All the fundamental amounts that represent the state information of the system are discrete. In principle, an integer can always be an exact representation of every such amount. For example, there are always an integral number of neutrons in a particular atom. Therefore, configurations of bits, like the binary digits in a computer, can correspond exactly to the most microscopic representation of that kind of state information.

The locations need to be connected, in a fast way, and a huge amount of data of all types be broadcasted, thus the digital world is launched a new era of human meeting across business, society and in every aspect of the lives. Never before culture destine the human as today, because the small city of world, communicate in mail, transfer money, chat by voice, or by video call, purchase a product ,to be delivered next, send the requested fee, and read as you want by the original language or translated version in a few minutes.

Further the highest powerful influence on human behavior, the effects of this shift on society are tremendous and, in particular, are dramatically changing our leadership responsibilities whether in politics, professionals in business, teachers in school or parents raising children.

The opportunity for users to accomplish the need for social interaction, entertainment and learning is almost equal to the risk of impairment to cognitive, emotional and behavioral development and even mental health issues. Digital technology is extremely powerful, and leaders have a heightened sense of responsibility to answer the call to action.

In the past, the information was spread by media contained a wide, range of content that viewers could use to obtain a full view of the news. Today, that is no longer the case and customers now request content to match their world view. The results are a new world where opinion is fact and the truth is debatable. And the concept of trust is now earned largely by collective experience rather than the symbols of faith.

\section{The philosophy of software - Computer Science ${ }^{[7]}$}

The philosophy of computer science is carry about the ontological, methodological, and ethical topics that rise from academic plan of computer science, and within the preparation of software development. Thus, the philosophy of computer science has the similar goals as the goals philosophy of other subfield of science, such the philosophy of biology or philosophy of the social sciences. In addition the philosophy of computer science studies the analysis of computational software, that is, human-made developing systems, which include the stages of cycle, design, specification, programming, verification, implementation, and testing of those systems.

\section{Philosophy of programming}

It is hard to see the connection between Philosophy and Programming. Because Philosophy is simply broader concept, Programming is a discipline, and philosophy is not a discipline. Also, Philosophy is about 
ideas and doing as much as you can with a limited knowledge base. That is the principle of philosophy, dealing with incomplete knowledge.

Much like mathematics, programming is a logical deductive system, it is just making is logical deductive system, and there is no philosophy, because everything is well-known. Is there philosophy in playing chess? Not everything is known in chess, because it is computationally too intensive. Also in programming not everything is known. Thus, I didn't compose all the libraries that I use in Java, and I have to make assumptions about the behavior of the libraries that I didn't compose; further this is the most important philosophy idea in programming the incomplete information, take example if you are older than 18 you can practice driving if no go back when you are 18. Does the Programming make arguments; does it express about anything inside the world; does it describe anything, only what to do, it uses data; moreover it can't refine data without added input. Programs do not create data, they can gather it or request it and analyze it; they cannot manufacture it. The data comes from the real world, not from programs, right? Go ahead; programming and philosophy have the follow similarities:

1) Programming is art of thinking and expressing, the same as when comparing with a philosophical problem, for example, when writing a philosophical paper or defending an argument or reasoning. Thinking and expressing the nature of anything is a philosophical mission.

2) The nature of computation is very close to the nature of the universe. The logical constraints of thought that the computer undertakes are similar to the rules of thought that a human uses.

3) Programmers can philosophize when developing software as they want, but at the end stage, when they publish it, there is an argument, if the software itself is philosophical in nature. However, also the philosophical paper is arguable, if it is philosophical in nature when it is published.

\section{Philosophy of Network}

Network offers the opportunity to interview of academy users and build their relationship. This isn't the sum of my networking philosophy, but it is the biggest thing the user achieved.

The academic field of analysis that studies this is called social network analysis. There is necessity to build network allows people to connect via social media, mobile, and email.

\section{Computer ethical philosophy}

Computer ethical philosophy concerned the behaviors of computer professionals regarding professional and social conduct, computer professionals responsibility relate to the computer technology usage categorize into three classes :

1. Programmer's personal codes and creations and their copyright.

2. Privacy of collected personal information and other database content conduct with the usage.

3. The influence of technology in society. 


\section{Software Neutral}

Generally, a key of business philosophy is evaluating and recommending best-fit EMIS (Information Management System (EMIS) space ) solutions and implementing software for the clients that supporting Environmental, Health, and Safety (EHS) .

\section{Philosophy of Secure Software (Privacy)}

Privacy is the facility of a user or group to isolate themselves, or information about themselves, and thus express themselves to specific networks. Once knowledge is private to users, it usually means that knowledge is inherently special for them. The domain of privacy overlays the security confidentiality related to each one, the domain of each one contains the permitions and the rights of use, as in addition the protection of information.

\section{Informational}

Data privacy states the developing relationship between technologies and the legal right to, announces the sharing of data about each item. Further, in addition to the issue of who is given access to information, there is other issues comprise whether a user has any permission rights to publish them, and/or the right to view, verify, and test that data.

\section{Philosophy of Internet}

The Internet conveys new worries where computers store some records of online photo, status update, Twitter post and personal blog, about each subscriber; moreover these data can be stored forever.

All knowledge is shared for researchers, and there are search engines, social-networking sites, photo/videosharing sites, personal web sites and blogs, and social media applications as Facebook offered to the users everywhere at the globe.

\section{Attractive Software}

Client focused on the design of software, always there is a question, if the software is attractive to the clients, because of that the developers focus on the software appearance. The organizations are passionate about the appearance and it is core to the business approach. The organization's philosophy and consulting approach for all services, including system designs, is to put the needs of the clients first. This client focused approach brings value to the client's organization and is a big reason why developer is selected to provide design services.

\section{Protect Your Own Computer}

Viruses and malware pose big problem to all computer users, especially if the used machine is not protected.

The computers are infected from networks usage some examples of attack are:

- Using Email: opening an infected email attachment or clicking on a spam link to a web page

- Surfing the Net: downloading software from malware web sites or clicking on links on those webpages

- Storing Data: opening files on an infected USB stick or removable hard drives 
A posed philosophy to protect your machine either your machine is online or offline to protect your data and devices.

\section{Conclusions}

The computer has effects in daily life of all domains, philosophy of computer science divide into several subdivisions first hardware or the necessary parts to be on, secondly the important software in order to achieves the goals of using computing, thirdly language to program application, fourth interaction patterns as step to design acceptable application, fourth networks to interact, fifth internet to post and share, sixth security to share in confidentiality, at the end anti-virus to protect the works.

\section{References}

[1] Philosophy.

(n.d.).

Retrieved

from

url:

http://www.port.ac.uk/research/csseresearchgroup/philosophy

[2] Rapaport, W. J. (March 4, 2017). Philosophy of Computer Science, Department of Philosophy and Department of Linguistics University at Buffalo. The State University of New York:

Department of Computer Science and Engineering March 4, 2017 Retrieved from the web site

[3] url: http://www.cse.buffalo.edu/_rapaport/

[4] Connecting Computer Science, 2e by Greg Anderson, David Ferro and Robert Hilton Massachusetts and, a PhD in Science and Technology Studies from Virginia publish by Course Technology Cenguage Learning in January 14, 2010

[5] Timeline of Computer History by Membership of Computer History Museum, Shoreline Blvd. Mountain View, CA, web site Copyright (C) in 2017 Retrieved from the web site url: http://www.computerhistory.org/timeline/software-languages/

[6] The History of Computers by SoftChalk LessonBuilder@ in 2008 _url :http://www.ptc.des.edu/Moody/comphistory/

[7] Digital Philosophy essay by Edward Fredkin in 13 April 2013 url: http://www.digitalphilosophy.org

[8] The Philosophy of Computer Science by Edward N. Zalta (ed.), published Tue Aug 20, 2013; substantive revision Thu Jan 19, 2017_url http://www.plato.stanford.edu/archives/spr2017/entries/computer-science

[9] Programming Is Philosophy By Alex Mills publisher wiki in May 26, 2013_url : http://wiki.c2.com/?ProgrammingIsPhilosophy

[10] Computer ethics From Wikipedia, the free encyclopedia in June 2011_url : https://en.wikipedia.org/wiki/Computer_ethics

[11] Philosophy by Computer Science and Software Engineering research Group University of Portsmouth in 2012 url: http://www.port.ac.uk/research/csseresearchgroup/philosophy

[12] Information Privacy From Wikipedia, the free encyclopedia on 14 August 2017

[13] Abstraction in Computer Science Timothy Colburn and Gary Shute on 5 June 2007

[14] Philosophy of Computer Science: An Introductory Course WILLIAM J. RAPAPORT State University of New York at Buffalo 